\title{
Elevated EXO1 expression is associated with breast carcinogenesis and poor prognosis
}

\author{
Jingjing Liu ${ }^{1,2,3,4,5}$, Jin Zhang ${ }^{1,2,3,4,5}$
}

${ }^{1}$ 3rd Department of Breast Cancer, China Tianjin Breast Cancer Prevention, Treatment and Research Center, Tianjin Medical University Cancer Institute and Hospital, Tianjin, China; ${ }^{2}$ Key Laboratory of Breast Cancer Prevention and Therapy of Ministry of Education, Tianjin, China; ${ }^{3}$ Key Laboratory of Cancer Prevention and Therapy, Tianjin, China; ${ }^{4}$ Tianjin's Clinical Research Center for Cancer, Tianjin, China; ${ }^{5}$ Tianjin Medical University Cancer Institute and Hospital, National Clinical Research Center for Cancer, Tianjin, China

Contributions: (I) Conception and design: All authors; (II) Administrative support: All authors; (III) Provision of study materials or patients: J Liu; (IV) Collection and assembly of data: All authors; (V) Data analysis and interpretation: All authors; (VI) Manuscript writing: All authors; (VII) Final approval of manuscript: All authors.

Correspondence to: Jin Zhang, PhD, MD. Huanhuxi Road Tiyuanbei, Hexi District, Tianjin 300060, China. Email: zhangjintjmuch1@163.com.

Background: Breast cancer is the most common cancer and leading cause of cancer mortality in women worldwide. Exonuclease 1 (EXO1), a protein with 5' to 3' exonuclease and RNase H activity, could be involved in mismatch repair and recombination. This study aims to investigate the prognostic value of EXO1 in breast cancer and explore the association between EXO1 expression and breast carcinogenesis.

Methods: The data of 1,215 breast cancer susceptibility gene (BRCA) samples were obtained from The Cancer Genome Atlas (TCGA). Real-time quantitative polymerase chain reaction (RT-qPCR) further verified the elevated mRNA expression level of EXO1 in human BRCA cells MDA-MB231 compared with that in human breast epithelial cells MCF-10A. EXO1 copy number was proved to be correlated with its expression level. Besides, Kaplan-Meier analysis, differentially expressed genes and function enrichment analysis were performed.

Results: Analysis of data from The Cancer Genome Atlas (TCGA) revealed that the EXO1 expression level in breast cancer tissues was significantly increased. Real-time quantitative polymerase chain reaction (RT-qPCR) supported the elevated mRNA expression level of EXO1 in human breast cancer cells MDA-MB231 compared with that in human breast epithelial cells MCF-10A. EXO1 copy number was shown to be correlated with its expression level. Kaplan-Meier analysis showed that elevated EXO1 was an indicator of poor breast cancer prognosis. Furthermore, differentially expressed genes and function enrichment analysis indicated that the cell cycle pathway and cardiac muscle contraction pathway were activated and inhibited respectively in breast cancer samples with high EXO1 expression.

Conclusions: Therefore, this study shows that elevated EXO1 expression is associated with carcinogenesis and poor prognosis in breast cancer, and might be a biomarker for breast cancer treatment.

Keywords: Breast cancer; exonuclease 1 (EXO1); prognosis; cell cycle pathway; cardiac muscle contraction pathway

Submitted Nov 03, 2020. Accepted for publication Jan 22, 2021.

doi: 10.21037/atm-20-7922

View this article at: http://dx.doi.org/10.21037/atm-20-7922

\section{Introduction}

Breast cancer is the most common cancer worldwide and accounts for the majority of cancer-related deaths in women (1). In 2012, there were 1.7 million new cases of breast cancer diagnosed, along with 198,000 breast cancer deaths reported in developed regions and 324,000 deaths in less developed regions (2). The etiology of breast cancer includes high-fat dietary intake, alcohol consumption, 
exposure to ionizing radiation, hormone use, oral contraceptives, in addition to other potential causes (3). The main curative therapies consist of antiestrogens, surgery, hormonal therapy, radiation therapy, and chemotherapy, which are accompanied with unpleasant side effects (1). Despite advancements in treatment, it has been difficult to establish improvements in survival of patients with recurrent or metastatic breast cancer (4). Consequently, expectations around breast cancer treatment and prognosis remain guarded. Given the World Health Organization suggests early detection improves outcomes and survival in breast cancer (1), it is necessary and important to find effective biomarkers for early diagnosis and prognosis of breast cancer.

Exonuclease 1 (EXO1), a protein with $5^{\prime}$ to $3^{\prime}$ exonuclease and $\mathrm{RNase} \mathrm{H}$ activity, could interact with mutator S homolog 2 (MSH2) and be involved in mismatch repair and recombination $(5,6)$. Previous studies revealed that EXO1 played a key role in induction of DNA damage checkpoints and DNA damage repair $(7,8)$, as a guardian of our genome to reduce cancer progression. Conversely, EXO1 is associated with cancer carcinogenesis and progression. This has been demonstrated in ovarian cancer, where EXO1 expression has been shown to be up-regulated suggesting a relationship with chemoresistance of ovarian cancer (9). Additionally, the allele of EXO1 K589E has shown an association with susceptibility to and development of lung cancer (10) and EXO1 K589E polymorphism has been associated with risk of cervical cancer and potential promotion of carcinogenesis of cervical cancer (11). Clinical data in Memorial sloan-kettering cancer center (MSKCC) and TCGA was revealed that the EXO1 was markedly related to the progression and prognosis in prostate cancer (12). Despite such evidence, to our knowledge the mechanism role of EXO1 in breast cancer has not been thoroughly investigated.

Bioinformative and experimental analyses were undertaken to explore the role of EXO1 as a biomarker in breast cancer. This study comprehensively analyzed the expression spectrum of EXO1 in a large cohort of breast cancer samples from The Cancer Genome Atlas (TCGA), and validated its elevated mRNA expression level in human breast cancer cell lines and clinical tissues using real-time quantitative polymerase chain reaction (RT-qPCR) and western blotting. Gene copy number varies in different diseases and its increase and deletion lead to altered expression levels of cancer-related genes (13). Epigenetic modifications are able to influence gene expression without altering nucleotide sequence, and DNA methylation is considered as a typical epigenetic modification (14). It is recognized that multiple genomic characteristics-including gene copy number, mRNA expression and methylationcan provide a systematic approach to research pertaining to the genomic landscape of cancers (13). Therefore, we also investigated the association of EXO1 expression with gene copy number and methylation to explore the EXO1 expression and affecting factors through multiomics approaches. Survival analysis and Cox regression analysis were performed to explore the prognostic value of EXO1 in breast cancer. Moreover, we conducted functional enrichment analysis to investigate the underlying mechanisms and biological processes in breast cancer.

We present the following article in accordance with the REMARK reporting checklist (available at http://dx.doi. org/10.21037/atm-20-7922).

\section{Methods}

\section{Study population}

The data of 1,215 breast cancer samples were obtained from TCGA (www.cancergenome.nih.gov). There were 1,102 tumor tissue samples and 113 adjacent tissue samples with gene copy number and data of methylation. Of these, 1,086 tumor tissue samples had complete information of tumor stage, and 1,097 tumor tissue samples had complete information of overall survival (OS) and survival status. Patient samples included 3 normal health and 4 breast cancer patients which were from Tianjin Medical University Cancer Institute and Hospital. All procedures performed in this study involving human participants were in accordance with the Declaration of Helsinki (as revised in 2013). All informed consents were obtained and our research was approved by Tianjin Medical University Cancer Institute and Hospital Ethical Committee.

\section{Differential expression analysis}

The edge R Bioconductor package was used to screen for differentially expressed genes (DEGs). Genes with average counts less than 10 in the samples were removed. Thresholds for significantly differential expression were set as follows: FDR $<0.05$ and $\mid \log 2 \mathrm{FCl}>1$ (FDR: False Discovery Rates; FC: Fold Change). 
Table 1 The primer sequences of RT-qPCR

\begin{tabular}{ll}
\hline Gene & Sequence \\
\hline EXO1 & F: 5'-GCAACTTCTTCGTGAGGGA-3' \\
& R: 5'-AGGAAGGTATTGTTGGCCCG-3' \\
GAPDH & F: GGTGAAGGTCGGTGTGAACG \\
& R: CTCGCTCCTGGAAGATGGTG \\
\hline
\end{tabular}

RT-qPCR, real-time quantitative polymerase chain reaction; $F$, forward; R, reverse.

\section{Function enrichment analysis}

The Database for Annotation, Visualization and Integrated Discovery (DAVID) (https://david.ncifcrf.gov/) analysis was conducted, and $\mathrm{P}$ value $<0.05$ was used as a threshold for statistical significance of enriched pathways.

\section{Cell culture}

The human breast cancer cell lines (MDA-MB-231, SKBR3, MCF-7, T47D) and human breast epithelial cell lines (MCF10A and 76N-Tert) were purchased from the American Type Culture Collection (Manassas, VA, USA). Besides, human breast cancer cell line Cal51 was from the German Collection of Microorganisms and Cell Cultures (Leibniz Institute DSMZ, Braunschweig, Germany). Breast cancer cell lines were cultured in RPMI 1640 medium (GIBCO, USA) supplemented with 10\% fetal bovine serum (Hyclone, USA). Breast epithelial cell lines were cultured in DMEM/F12 medium (GIBCO, USA) containing amino acid mixture (L-Alanine, L-Asparagine, L-Aspartic Acid, L-Glutamic Acid, L-Glycine, L-Proline, and L-Serine), HEPES $(5 \mathrm{~mL})$, insulin $(500 \mu \mathrm{L})$, hydrocortisone $(500 \mu \mathrm{L})$, epidermal growth factor $(250 \mu \mathrm{L})$, and penicillinstreptomycin $(5 \mathrm{~mL})$. The cells were incubated in an incubator (Thermo Forma, USA) at $37{ }^{\circ} \mathrm{C}$ with $5 \% \mathrm{CO}_{2}$.

\section{Real-time quantitative polymerase chain reaction (RT-qPCR)}

Total RNA was extracted from cells using chloroform. The integrity of RNA samples was determined by $1 \%$ (weight/ volume) agarose gel electrophoresis and the concentration was measured by NanoDrop ND-2000 spectrophotometer (NanoDrop Technologies, USA). RNA was then reversely transcribed into cDNA according to the instructions of PrimeScript $^{\mathrm{TM}}$ RT Master Mix kit (TakaRa, China). RT-
qPCR was performed using the SYBR Premix Ex Taq kit (Roche, Basel, Switzerland) on CFX96 Bio-Rad RealTime PCR System (Bio-Rad, USA). The reaction system included $10.0 \mu \mathrm{L} 2 \times$ SYBR Premix Ex Taq, $1.0 \mu \mathrm{L}$ upstream primer, $1.0 \mu \mathrm{L}$ downstream primer, $1 \mu \mathrm{L}$ cDNA, and $7 \mu \mathrm{L}$ RNase-free $\mathrm{dH}_{2} \mathrm{O}$. The primer sequence for RT-qPCR is shown in Table 1. The experimental data was analyzed using $2^{-\Delta \Delta C t}$ method with GAPDH as an endogenous control. The sequences of primers in this research were shown in the Table S1.

\section{Western blotting}

Total proteins were separated, and western blotting was conducted and repeated several times as previous standard method (15). Antibody against EXO1 (YT1646, 1:2,000, Texas, USA) and GAPDH (YM3215, 1:5,000, Texas, USA) antibody were both from Immunoway, and GAPDH as a control. Anti-rabbit Ig secondary antibody (1:2,000, CA, USA) was purchased from Vector Laboratories.

\section{Statistical analysis}

The associations between EXO1 expression and clinicopathologic variables in breast cancer were analyzed by using a Chi-square test. A two-sided t-test was conducted to analyze the association between EXO1 expression and age, and compare the EXO1 expressions in tumor tissues and adjacent tissues. The Kaplan-Meier method was applied to estimate the OS, and comparisons of OS among multiple groups were conducted using a log-rank test. Pearson correlation was conducted to analyze the relationship between EXO1 expression and methylation. The t-test compared the difference of mRNA expression levels between the experimental and control groups in RT-qPCR and western blotting. In the analysis of EXO1-related genes, the tumor tissue samples were divided into two groups using the median of EXO1 expression level as the threshold, with abbreviations "EXO1_high" and "EXO1_ low". A P value $<0.05$ was considered statistically significant in all statistical analyses.

\section{Results}

\section{Correlation of EXO1 expression with clinicopathologic features in breast cancer patients}

Tumor tissue samples were divided into "EXO1_high" and 
Table 2 The association between EXO1 expression and demographic and clinicopathological parameters of breast cancer patients

\begin{tabular}{|c|c|c|c|c|}
\hline Parameters & \multicolumn{2}{|c|}{ EXO1 expression } & $\chi^{2}$ & $P$ value \\
\hline Age, years (mean \pm SD) & $57.87 \pm 13.31$ & $60.37 \pm 12.88$ & & 0.001709 \\
\hline Gender & & & 0.76384 & 0.3821 \\
\hline Female & 540 & 545 & & \\
\hline Classification of breast cancer & & & 7.751 & 0.1011 \\
\hline $\operatorname{ER}(+) \operatorname{PR}(+) H E R 2(-)$ & 191 & 214 & & \\
\hline $\mathrm{ER}(+) \mathrm{PR}(+) \mathrm{HER} 2(+)$ & 52 & 60 & & \\
\hline ER(-)PR(-)HER2(+) & 22 & 19 & & \\
\hline Radiotherapy & & & 0.81581 & 0.665 \\
\hline Yes & 352 & 365 & & \\
\hline No & 150 & 172 & & \\
\hline Unknown & 30 & 28 & & \\
\hline Chemotherapy & & & 5.7512 & 0.05638 \\
\hline Yes & 303 & 280 & & \\
\hline No & 297 & 211 & & \\
\hline Unknown & 2 & 4 & & \\
\hline Alive & 458 & 486 & & \\
\hline
\end{tabular}

"EXO1_low" groups using the median of EXO1 expression level as threshold. The associations between EXO1 expression and OS status, tumor stage, and gender, were analyzed using a Chi-square test. There was no significant difference in gender $(\mathrm{P}=0.3821)$ and tumor stage $(\mathrm{P}=0.2383)$ between groups. Patients with high EXO1 expression showed a tendency towards death $(\mathrm{P}=0.02272)$. The association between EXO1 expression and age was analyzed using a two-sided t-test. Age was lower in the "EXO1_ high" group compared with that in the "EXO1_low" group ( $\mathrm{P}=0.001709$, Table 2).

\section{High expression of EXO1 indicated poor prognosis in} breast cancer patients

Kaplan-Meier method and log-rank test were conducted to estimate and compare OS among multiple groups. The OS in the "EXO1_high" group was significantly worse than that in the "EXO1_low" group $(\mathrm{P}=0.012)$ (Figure 1A). OS analysis was also conducted with gender and EXO1 expression level as variables simultaneously. For patients in the same age group, OS of patients with low EXO1 expression was superior $(\mathrm{P}<0.0001)$ (Figure 1B), indicating $\mathrm{EXO1}$ as an independent risk factor for breast cancer. 
A

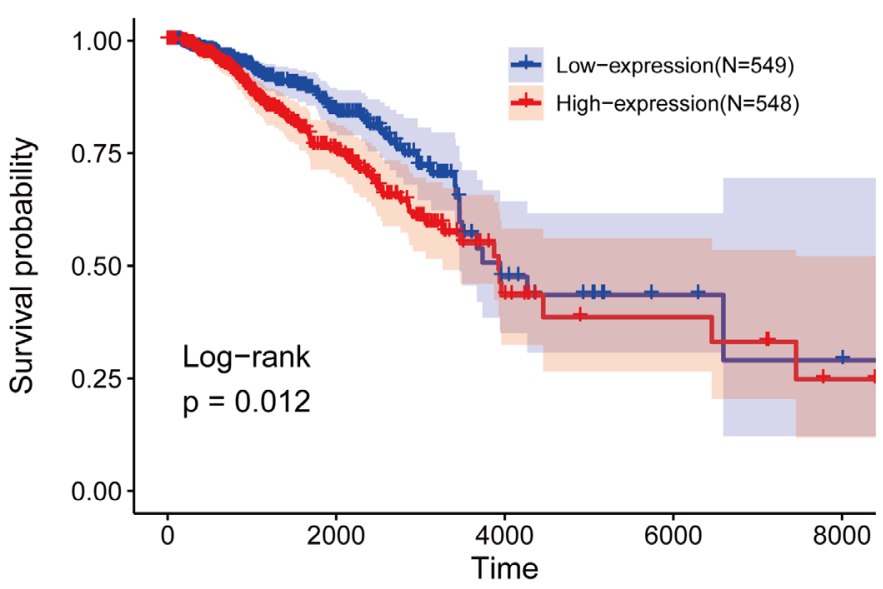

Number at risk

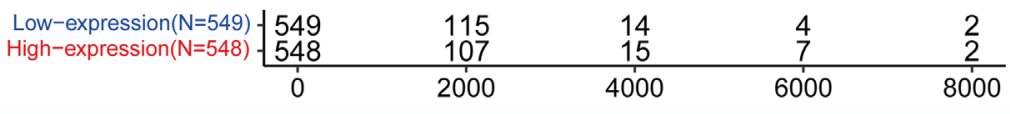

B

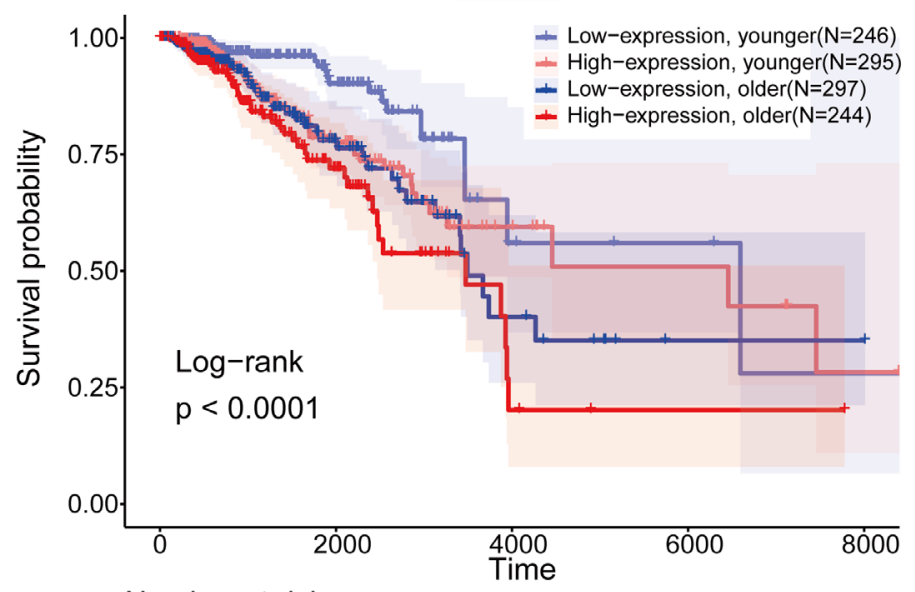

Number at risk

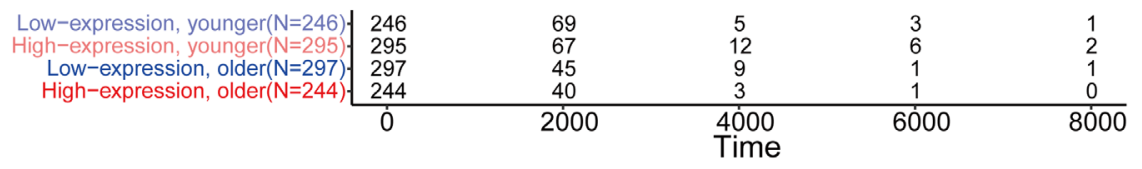

Figure 1 Elevated expression of EXO1 was associated with poor prognosis in breast cancer patients. (A) The overall survival (OS) in EXO1_ high group was inferior compared with that in EXO1_low group ( $\mathrm{P}=0.012)$; (B) the younger patients showed superior OS compared with the older patients. The OS of patients with high EXO1 expression was adverse for the patients in the same age group $(\mathrm{P}<0.0001)$.

\section{EXO1 expression was increased in breast cancer tissues}

The average expression level of EXO1 in breast cancer tissues was 9.34 compared to 5.83 seen in adjacent tissues. The EXO1 expression in breast cancer tissues was significantly higher than that in adjacent tissues $(\mathrm{P}=0)$ (Figure 2A). Although significant differences in EXO1 expression levels were observed between adjacent tissues and tumor tissues of different stages $(\mathrm{P}=0)$, EXO1 expression was not significantly correlated with tumor stage (adjacent tissues: 5.83, stage I: 8.79, stage II: 9.48, stage III: 9.45, stage IV: 9.42, stage V: $8.80 ; \mathrm{P}=0.419$ ) (Figure $2 B$ ). These results showed EXO1 might play a key role in breast carcinogenesis with high expression level in the early stage. 


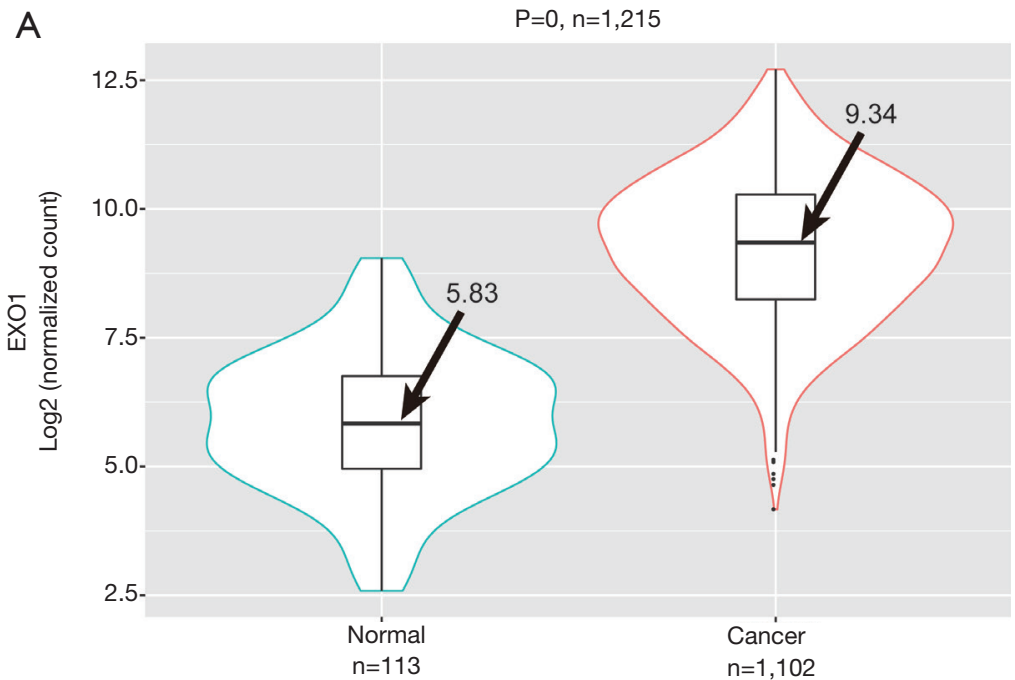

B

$P=0, n=1,199$

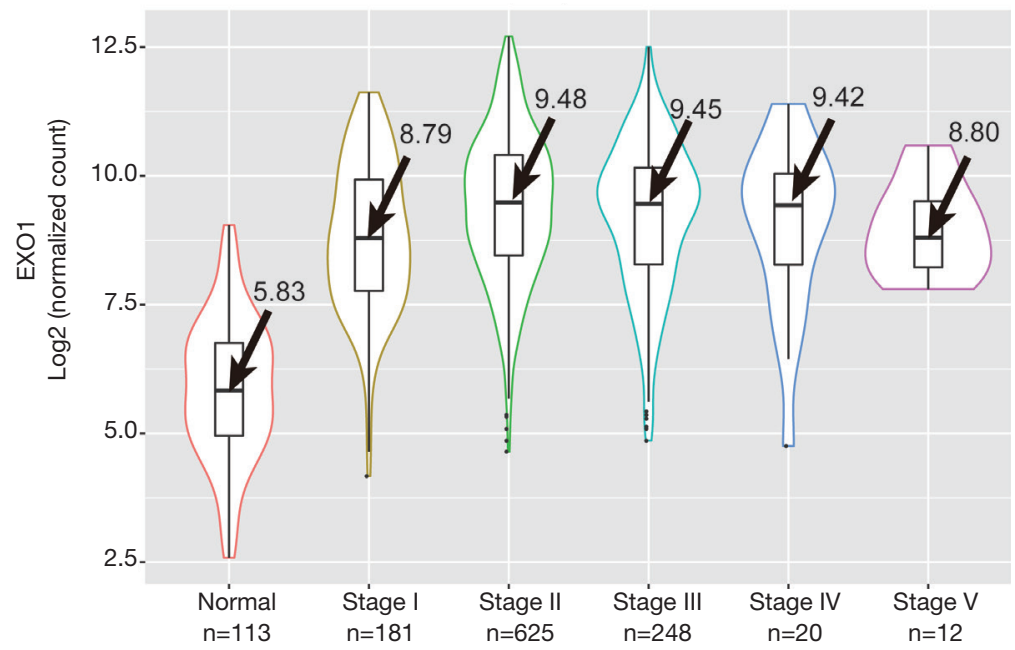

Figure 2 Elevated EXO1 expression level was associated with breast cancer carcinogenesis. (A) The breast cancer tissues showed increased EXO1 expression level compared with that in adjacent tissues $(\mathrm{P}=0)$; (B) there were significant differences in EXO1 expression levels between adjacent tissues and tumor tissues of different stages $(\mathrm{P}=0)$, however, EXO1 expression level was not correlated with tumor stage $(\mathrm{P}=0.419)$.

\section{Validation of elevated EXO1 expression in cell lines and tissues}

In order to verify that EXO1 expression was increased in breast cancer, RT-qPCR and western blotting were used to determine the mRNA (Figure $3 A$ ) and protein expressions (Figure $3 B$ ) of EXO1 in human breast cancer cell lines and human breast epithelial cell lines. Besides, the clinical tissues, including tumor and normal tissues, were used to confirmed the high expression of EXO1 in breast cancer (Figure 3C). The results revealed that the mRNA and protein expression of EXO1 in cells and tissues were significantly higher in breast cancer.

\section{Correlations of EXO1 expression with gene copy number and methylation}

DNA copy number variation has been found to feature in most cancers. In this study, we investigate the association between EXO1 gene copy number and its expression level. DNA methylation was previously shown to be involved in tumorigenesis (16). This study analyzed the association between EXO1 expression and its methylation, 
A

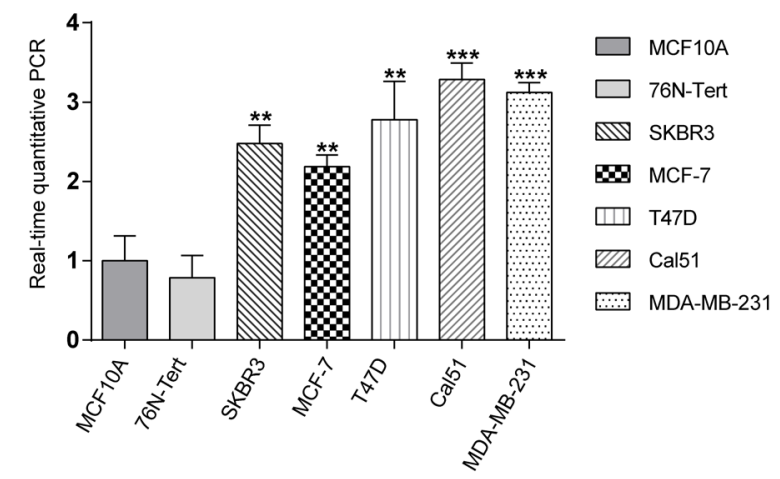

B

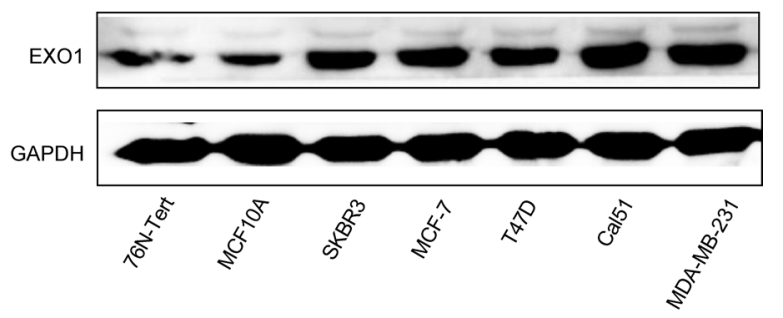

C



Figure 3 EXO1 expression verification in cell lines and tissues. (A) mRNA expression in human breast epithelial cell lines and breast cancer cell lines; (B) protein expression in human breast epithelial cell lines and breast cancer cell lines; (C) protein expression in normal and breast cancer tissues. ${ }^{* *} \mathrm{P}<0.01,{ }^{* *} \mathrm{P}<0.001$, vs. MCF10A group.

with Pearson correlation analysis showing no significant correlation between EXO1 expression and its methylation $(\mathrm{P}=0.6597, \mathrm{r}=-0.015)$ (Figure $4 A$ ). The focal segment score of each gene was calculated using GISTIC in TCGA, which represented changes of copy number. The " -1 ", "0", and "1" were used to represent gene copy number loss, normal copy number, and copy number amplification respectively. The EXO1 expression level in group " 1 " was significantly higher than that in groups " -1 " and " 0 " (group " -1 ": 9.03, group "0": 9.14, group "1": 10.13; $\mathrm{P}=4.66 \mathrm{e}-06)$ (Figure 4B).

\section{The molecular mechanism of EXO1 underlying breast cancer carcinogenesis and poor prognosis}

To explore the underlying molecular mechanism of EXO1 in breast cancer carcinogenesis, we screened the DEGs between "EXO1_high" and "EXO1_low" samples. As shown in https://cdn.amegroups.cn/static/public/ATM20-7922-1.xlsx, a total of 1,593 DEGs were obtained. The expression heatmap of the DEGs is shown in Figure 5 A. The up-regulated genes were mainly distributed in the pathways including cell cycle, oocyte meiosis, and neuroactive ligandreceptor interaction (Figure 5B), with the cell cycle pathway showing highest significance. The down-regulated genes were mainly distributed in the pathways including cardiac muscle contraction, neuroactive ligand-receptor interaction, and peroxisome proliferator-activated receptors (PPAR) (Figure 5C), with the cardiac muscle contraction pathway showing highest significance. The cell cycle pathway of the up-regulated genes is shown in Figure $6 \mathrm{~A}$ with red color indicating up-regulated genes and light green color indicating subpathways. The cardiac muscle contraction pathway the down-regulated genes was shown in Figure $6 B$ with dark green color indicating down-regulated genes and light green color indicating subpathways.

\section{Discussion}

Breast cancer is the main cause of cancer mortality in women and is considered a global public health issue $(17,18)$. Current prognostic factors of breast cancer include clinical stage, tumor size and stage, axillary nodal status, lymphovascular involvement, and hormone receptor status, with numerous ongoing efforts to evaluate new prognostic factors in breast cancer risk (19). Gene-expression profiling provides the possibility of accurate prognostic information, which plays a significant role in selecting optimal therapy for individuals and avoiding over- and under-treatment (20). 

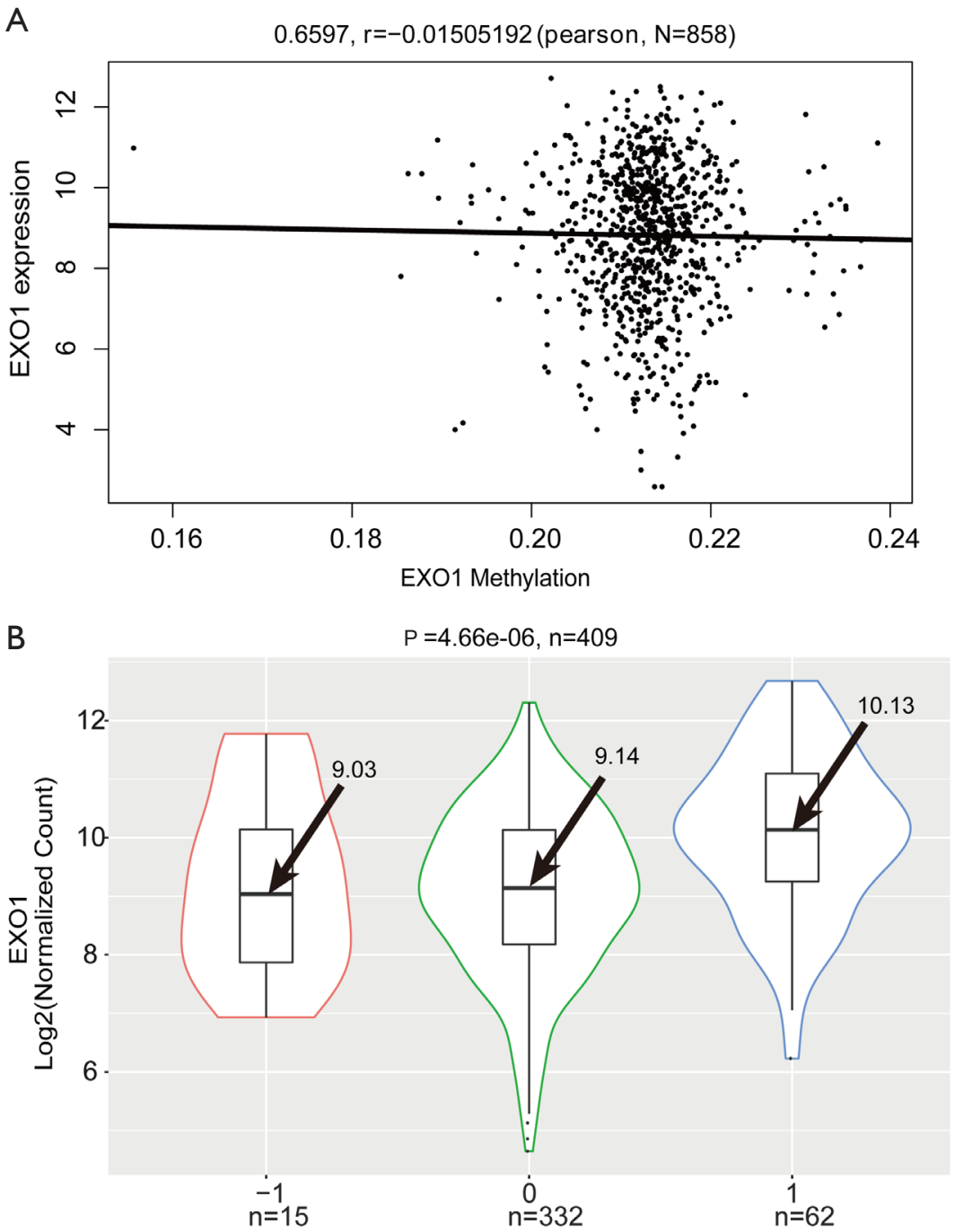

Figure 4 EXO1 expression was positively correlated with gene copy number, while it was not correlated with methylation values. (A) There was no significant correlation between EXO1 expression and its methylation ( $\mathrm{P}=0.6597, \mathrm{r}=-0.015)$; (B) elevated EXOI expression was observed in the group with gene copy number amplification compared with the groups with gene copy number loss and normal copy number $(\mathrm{P}=4.66 \mathrm{e}-06)$.

This study showed EXO1 expression level was increased in breast cancer tissues compared with that in adjacent tissues, and high expression level in the early stages of breast cancer. The elevated expression of EXO1 was validated in human breast cancer cell line MDA-MB231 by RT-qPCR. This study also found that patients with high expression level of EXO1 had inferior OS compared to patients with low expression level of EXO1. These results indicated EXO1 might be a potential biomarker for breast cancer carcinogenesis and prognosis.

EXO1 is an exonuclease associated with multiple
DNA metabolic processes such as DNA replication and DNA repair of mismatch and double-stranded break (21). Although the relationships between EXO1 dysfunction and several cancers have been confirmed, the specific mechanism varies between cancers (22). Mutations of the nuclease domain or the binding domains of MLH1 and MSH2 in EXO1 could contribute to cancer risks through loss of protein function (22-24), while elevated expression of EXO1 might be involved in cancers through increasing genomic instability. Valente $\mathrm{V}$ et al. found that EXO1 was upregulated in astrocytomas, and EXO1 silence contributed 
A



Class

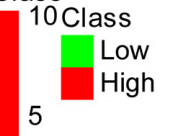

B

Activated

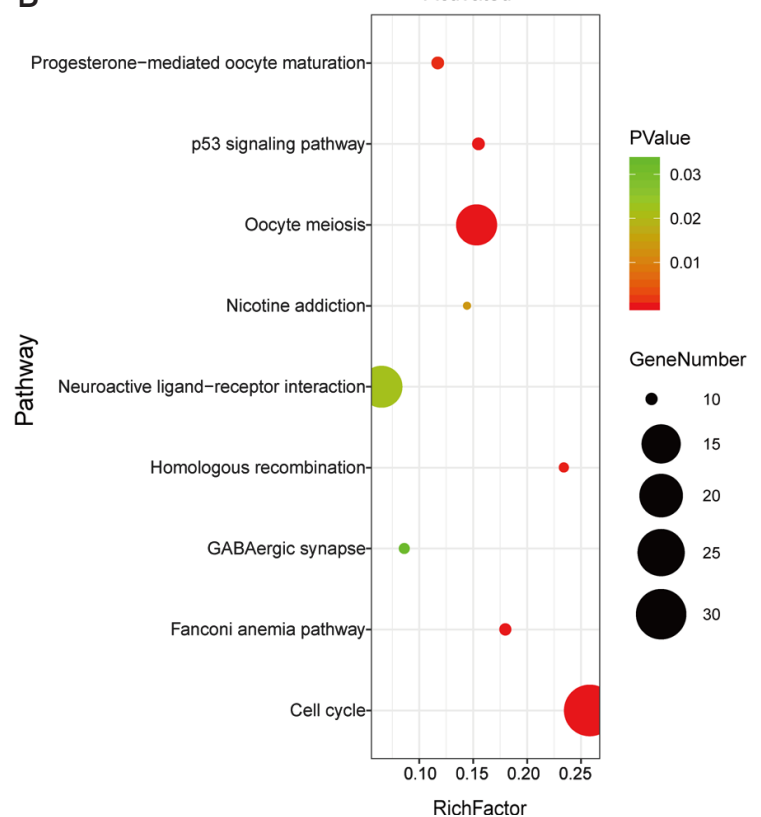

C

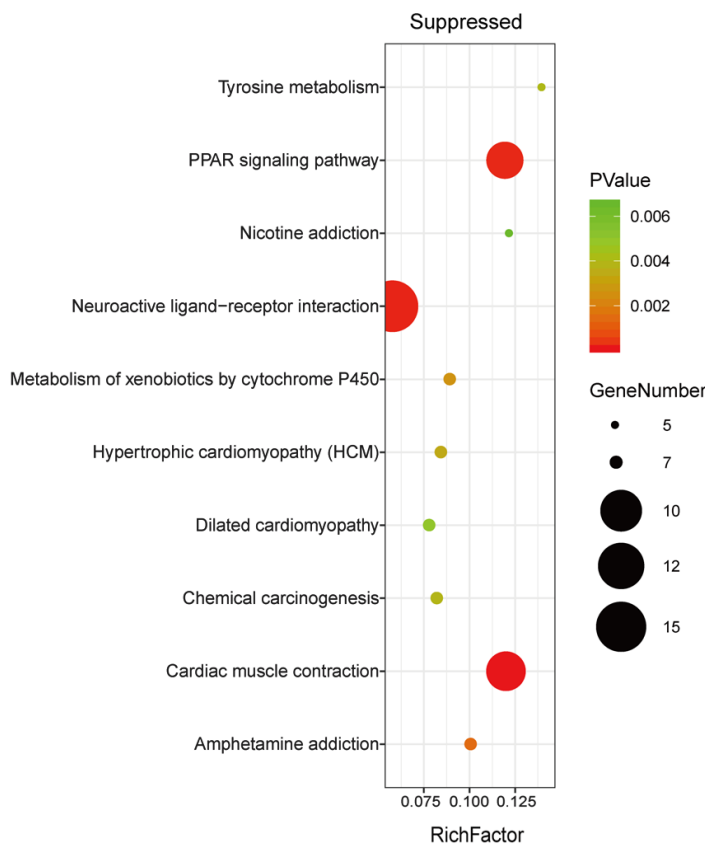

Figure 5 Elevated EXO1 expression might mediate breast cancer carcinogenesis and poor prognosis by targeting several pathways. (A) The expression heatmap of 1,593 differentially expressed genes (DEGs) in EXO1_high and EXO1_low groups; (B) Kyoto encyclopedia of genes and genomes (KEGG) pathways enriched by up-regulated genes. The horizontal axis represented the enrichment ratio, and the vertical axis represented the enriched pathway. The size of the circle corresponded to the number of enriched genes, and the color represented $\mathrm{P}$ value; (C) KEGG pathways enriched by down-regulated genes. The horizontal axis represented the enrichment ratio, and the vertical axis represented the enriched pathway. The size of the circle corresponded to the number of enriched genes, and the color represented $\mathrm{P}$ value. 
A

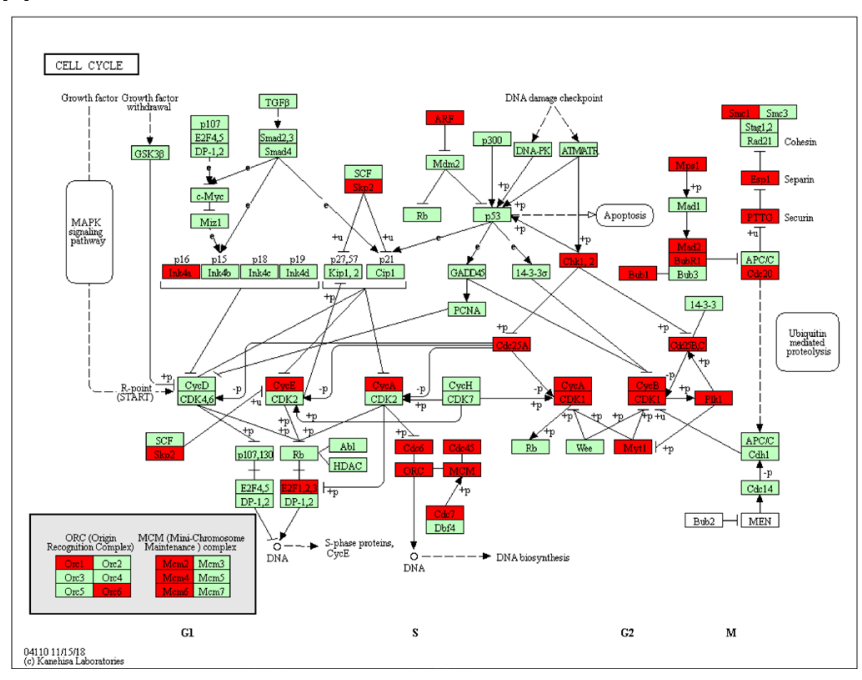

B



Figure 6 The cell cycle pathway showed the most significant upregulation while the cardiac muscle contraction pathway showed the most significant downregulation in EXO1_high breast cancer patients. (A) The pathway mapping plot of cell cycle pathway. The red color indicated up-regulated genes and light green color indicated subpathways of cell cycle pathway; (B) The pathway mapping plot of cardiac muscle contraction pathway. The dark green color indicated down-regulated genes and light green color indicated subpathways of cardiac muscle contraction pathway.

to faster double-strand breaks repair (25). Muthulakshmi et al. suggested increased EXO1 expression was related to increased unrectified DNA repairs and genomic instability in breast cancer (26). It is known that genomic instability is a characteristic seen in most cancers and has been observed in breast cancer (27-29). Thus, it is inferred that elevated EXO1 might be involved in carcinogenesis and poor prognosis of breast cancer by increasing genomic instability.

A previous study revealed DNA copy number exerted a direct effect on gene expression in breast cancer, including tumors and the corresponding cell lines (30). The alteration of DNA copy number can result in gene expression deregulation directly and might promote cancer development and progression. Furthermore, DNA methylation can also alter the expression level of genes (14). To explore the EXO1 expression and affecting factors, this study also investigated the association between EXO1 expression and gene copy number and methylation, showing EXO1 expression in the group with gene copy number amplification was significantly elevated compared to the groups with gene copy number loss and normal copy number. This was also consistent with existing research; however, there was no significant correlation between EXO1 expression and its methylation. Thus, it is speculated that EXO1 might exert its effects through integration of multi-omics effects.

EXO1 could regulate the cell cycle checkpoints lead to double-strand breaks, cell-cycle arrest, cell death, or cellular transformation (22), which interacted with multiple critical proteins, including 14-3-3 complex and 9-1-1 complex physically and functionally $(31,32)$. The cell cycle pathway was shown to be activated by CASC11 in gastric cancer (33). This pathway was also found to be involved in prostate cancer (34). In small cell lung cancer and nonsmall cell lung cancer, cell cycle upregulation was observed through activating pro-proliferative genes or inhibiting cell cycle inhibitor (35). The cell cycle pathway was associated with breast cancer prognosis and its upregulation might play a key role in promoting breast cancer progression (36). In this study, we found several pathways up-regulated in breast cancer samples with high EXO1 expression level, including cell cycle, oocyte meiosis, and neuroactive ligand-receptor interaction pathways, with the cell cycle pathway showing the most significant up-regulation. The expression levels of several cell cycle pathway-related genes were increased, including CDC25A, CycE, and CDK1. Elevated expression of CDC25A was correlated with poorer outcomes in breast cancer (37) while high levels of $\mathrm{CycE}$ were associated with reduced OS in breast cancer (38). Additionally, alteration of CDK1 was observed in breast tumor cases (39). Moreover, 
previous research indicated that the EXO1 over-expression activated RAS/AKT/MYC/E2F1 signaling pathways results in elevated cell proliferation, genomic instability (26). These results were consistent with our findings.

\section{Conclusions}

This study showed increased EXO1 expression was associated with carcinogenesis and poor prognosis in breast cancer, likely through regulating cell cycle or cardiac muscle contraction pathways. EXO1 might be an effective prognostic biomarker and novel therapeutic target for breast cancer; however, there are some limitations to this study. The association of EXO1 expression with suggested pathways-including cell cycle and cardiac muscle contraction pathways-were not experimentally validated and require further investigation in our follow-up study.

\section{Acknowledgments}

Funding: This work was supported by National Natural Science Foundation of China (grant number: 81672623); General project of scientific research program of Tianjin Municipal Education Commission (grant number: 2019KJ186); Tianjin Municipal Science and Technology Bureau (grant number: 19YFZCSY00030).

\section{Footnote}

Reporting Checklist: The authors have completed the REMARK reporting checklist. Available at http:// dx.doi.org/10.21037/atm-20-7922

Data Sharing Statement: Available at http://dx.doi. org/10.21037/atm-20-7922

Conflicts of Interest: Both authors have completed the ICMJE uniform disclosure form (available at http://dx.doi. org/10.21037/atm-20-7922). The authors have no conflicts of interest to declare.

Ethical Statement: The authors are accountable for all aspects of the work in ensuring that questions related to the accuracy or integrity of any part of the work are appropriately investigated and resolved. All procedures performed in this study involving human participants were in accordance with the Declaration of Helsinki (as revised in 2013). Our research was approved by Tianjin
Medical University Cancer Institute and Hospital Ethical Committee and all informed consents were obtained.

Open Access Statement: This is an Open Access article distributed in accordance with the Creative Commons Attribution-NonCommercial-NoDerivs 4.0 International License (CC BY-NC-ND 4.0), which permits the noncommercial replication and distribution of the article with the strict proviso that no changes or edits are made and the original work is properly cited (including links to both the formal publication through the relevant DOI and the license). See: https://creativecommons.org/licenses/by-nc-nd/4.0/.

\section{References}

1. Akram M, Iqbal M, Daniyal M, et al. Awareness and current knowledge of breast cancer. Biol Res 2017;50:33.

2. Ferlay J, Soerjomataram I, Dikshit R, et al. Cancer incidence and mortality worldwide: sources, methods and major patterns in GLOBOCAN 2012. Int J Cancer 2015;136:E359-86.

3. Wang HC, Chiu CF, Tsai RY, et al. Association of genetic polymorphisms of EXO1 gene with risk of breast cancer in Taiwan. Anticancer Res 2009;29:3897-901.

4. Giordano SH, Buzdar AU, Smith TL, et al. Is breast cancer survival improving? Cancer 2004;100:44-52.

5. Tran PT, Erdeniz N, Symington LS, et al. EXO1-A multi-tasking eukaryotic nuclease. DNA Repair (Amst) 2004;3:1549-59.

6. Eccleston J, Yan C, Yuan K, et al. Mismatch repair proteins MSH2, MLH1, and EXO1 are important for classswitch recombination events occurring in B cells that lack nonhomologous end joining. J Immunol 2011;186:2336-43.

7. Sperka T, Wang J, Rudolph KL. DNA damage checkpoints in stem cells, ageing and cancer. Nat Rev Mol Cell Biol 2012;13:579-90.

8. Bolderson E, Tomimatsu N, Richard DJ, et al. Phosphorylation of Exo1 modulates homologous recombination repair of DNA double-strand breaks. Nucleic Acids Res 2010;38:1821-31.

9. Zhou J, Wang Y, Wang Y, et al. FOXM1 modulates cisplatin sensitivity by regulating EXO1 in ovarian cancer. PLoS One 2014;9:e96989.

10. Hsu NY, Wang HC, Wang CH, et al. Lung cancer susceptibility and genetic polymorphisms of Exo1 gene in Taiwan. Anticancer Res 2009;29:725-30.

11. Luo X, Hong XS, Xiong XD, et al. A single nucleotide polymorphism in EXO1 gene is associated with cervical 
cancer susceptibility in Chinese patients. Int J Gynecol Cancer 2012;22:220-5.

12. Luo F, Wang YZ, Lin D, et al. Exonuclease 1 expression is associated with clinical progression, metastasis, and survival prognosis of prostate cancer. J Cell Biochem 2019.

13. Pinkel D, Segraves R, Sudar D, et al. High resolution analysis of DNA copy number variation using comparative genomic hybridization to microarrays. Nat Genet 1998;20:207-11.

14. Watson CT, Roussos P, Garg P, et al. Genome-wide DNA methylation profiling in the superior temporal gyrus reveals epigenetic signatures associated with Alzheimer's disease. Genome Med 2016;8:5.

15. Zhang JG, Zhou HM, Zhang X, et al. Hypoxic induction of vasculogenic mimicry in hepatocellular carcinoma: role of HIF-1 alpha, RhoA/ROCK and Rac1/PAK signaling. BMC Cancer 2020;20:32.

16. Issa JP. DNA methylation as a therapeutic target in cancer. Clin Cancer Res 2007;13:1634-7.

17. Martin-Sanchez JC, Cleries R, Lidon C, et al. Bayesian prediction of lung and breast cancer mortality among women in Spain (2014-2020). Cancer Epidemiol 2016;43:22-9.

18. Veronesi U, Boyle P, Goldhirsch A, et al. Breast cancer. Lancet 2005;365:1727-41.

19. Isaacs C, Stearns V, Hayes DF. New prognostic factors for breast cancer recurrence. Semin Oncol 2001;28:53-67.

20. Benson JR, Jatoi I, Keisch M, et al. Early breast cancer. Lancet 2009;373:1463-79.

21. Keijzers G, Liu D, Rasmussen LJ. Exonuclease 1 and its versatile roles in DNA repair. Crit Rev Biochem Mol Biol 2016;51:440-51.

22. Keijzers G, Bakula D, Petr MA, et al. Human Exonuclease 1 (EXO1) Regulatory Functions in DNA Replication with Putative Roles in Cancer. Int J Mol Sci 2018;20.

23. Sun X, Zheng L, Shen B. Functional alterations of human exonuclease 1 mutants identified in atypical hereditary nonpolyposis colorectal cancer syndrome. Cancer Res 2002;62:6026-30.

24. Schaetzlein S, Chahwan R, Avdievich E, et al. Mammalian Exo1 encodes both structural and catalytic functions that play distinct roles in essential biological processes. Proc Natl Acad Sci U S A 2013;110:E2470-9.

25. de Sousa JF, Torrieri R, Serafim RB, et al. Expression signatures of DNA repair genes correlate with survival prognosis of astrocytoma patients. Tumour Biol 2017;39:1010428317694552.

26. Muthuswami M, Ramesh V, Banerjee S, et al. Breast tumors with elevated expression of $1 \mathrm{q}$ candidate genes confer poor clinical outcome and sensitivity to Ras/PI3K inhibition. PLoS One 2013;8:e77553.

27. De Lange T. Telomere-related genome instability in cancer. Cold Spring Harb Symp Quant Biol 2005;70:197-204.

28. Melchor L, Benitez J. An integrative hypothesis about the origin and development of sporadic and familial breast cancer subtypes. Carcinogenesis 2008;29:1475-82.

29. Hu X, Stern HM, Ge L, et al. Genetic alterations and oncogenic pathways associated with breast cancer subtypes. Mol Cancer Res 2009;7:511-22.

30. Pollack JR, Sorlie T, Perou CM, et al. Microarray analysis reveals a major direct role of DNA copy number alteration in the transcriptional program of human breast tumors. Proc Natl Acad Sci U S A 2002;99:12963-8.

31. Chen X, Kim IK, Honaker Y, et al. 14-3-3 proteins restrain the Exo1 nuclease to prevent overresection. J Biol Chem 2015;290:12300-12.

32. Ngo GH, Lydall D. The 9-1-1 checkpoint clamp coordinates resection at DNA double strand breaks. Nucleic Acids Res 2015;43:5017-32.

33. Zhang L, Kang W, Lu X, et al. LncRNA CASC11 promoted gastric cancer cell proliferation, migration and invasion in vitro by regulating cell cycle pathway. Cell Cycle 2018;17:1886-900.

34. Lee JT, Lehmann BD, Terrian DM, et al. Targeting prostate cancer based on signal transduction and cell cycle pathways. Cell Cycle 2008;7:1745-62.

35. Coe BP, Lockwood WW, Girard L, et al. Differential disruption of cell cycle pathways in small cell and nonsmall cell lung cancer. Br J Cancer 2006;94:1927-35.

36. Liu J, Campen A, Huang S, et al. Identification of a gene signature in cell cycle pathway for breast cancer prognosis using gene expression profiling data. BMC Med Genomics 2008;1:39.

37. Evans KL. Overexpression of CDC25A associated with poor prognosis in breast cancer. Mol Med Today 2000;6:459.

38. Keyomarsi K, Tucker SL, Buchholz TA, et al. Cyclin E and survival in patients with breast cancer. N Engl J Med 2002;347:1566-75.

39. Peters MG, Vidal Mdel C, Gimenez L, et al. Prognostic value of cell cycle regulator molecules in surgically resected stage I and II breast cancer. Oncol Rep 2004;12:1143-50.

(English Language Editor: M. Hawkins)

Cite this article as: Liu J, Zhang J. Elevated EXO1 expression is associated with breast carcinogenesis and poor prognosis. Ann Transl Med 2021;9(2):135. doi: 10.21037/atm-20-7922 
Supplementary

Table S1 Primer sequences for RT-PCR.

\begin{tabular}{lllll}
\hline Genes & Forward Primer $\left(5^{\prime}-3^{\prime}\right)$ & Reverse Primer $\left(5^{\prime}-3^{\prime}\right)$ & Product & $\operatorname{Tm}\left({ }^{\circ} \mathrm{C}\right)$ \\
\hline EXO1 & GCAACTTCTTCGTGAGGGA & AGGAAGGTATTGTTGGCCCG & 537 & 58 \\
GAPDH & GGTGAAGGTCGGTGTGAACG & CTCGCTCCTGGAAGATGGTG & 233 & 58 \\
\hline
\end{tabular}

